\title{
Development of a functional HTS assay for the APJ receptor
}

Angela Giddings'

Scott Runyon ${ }^{2}$

James Thomas ${ }^{2}$

Julianne Tajuba'

Katherine Bortoff'

Rangan Maitra'

'Center for Pharmacology and Toxicology, ${ }^{2}$ Center for Organic and Medicinal Chemistry, Division of Discovery Sciences, RTI International, Research Triangle Park, NC, USA
Correspondence: Rangan Maitra

Center for Pharmacology and Toxicology,

Division of Discovery Sciences,

RTI International, 3040 Cornwallis Drive, P.O. Box 12194, Research Triangle Park,

NC 27709, USA

Tel $+|91954| 6795$

Fax +19195418868

Email rmaitra@rti.org
This article was published in the following Dove Press journal:

International Journal of High Throughput Screening

15 April 2010

Number of times this article has been viewed

\begin{abstract}
The apelin receptor (APJ) is rapidly emerging as an attractive therapeutic target for various diseases. To date, only one nonpeptide APJ agonist has been reported and new chemotypes having improved pharmacological properties are needed to expedite maturation of this receptor as a viable target. Therefore, the development of a high-throughput screening (HTS) assay for APJ should facilitate the discovery of novel ligands as in vivo probes of APJ function. In this report, an HTS compatible assay for the APJ is demonstrated. This assay takes advantage of functional coupling of APJ with the promiscuous $\mathrm{G \alpha q}_{16}$ protein and consequent mobilization of intracellular calcium, which can be measured in a microplate format using a fluorescent calcium probe. The assay was developed and validated using several known peptides derived from preproapelin. Most of the apelin peptides tested activated APJ at subnanomolar concentrations in accordance with their reported median effective concentration values. The assay was deemed robust and reproducible with a Z-factor of $\sim 0.6$ and tolerance to ethanol, dimethyl sulfoxide or a mixture of these two commonly used solvents to a final concentration of $1 \%$. The assay was further validated by screening a small library of compounds for APJ activity. A hit rate of $\sim 0.5 \%$ was achieved and confirmed by counter-screening against parental ${\mathrm{G} \alpha \mathrm{q}_{16}}_{1}$ cells lacking APJ. In conclusion, an HTS assay has been established to rapidly identify new ligands for the APJ. Novel APJ ligands having suitable drug-like properties are greatly needed to probe the in vivo pharmacology of this emerging therapeutic target.
\end{abstract}

Keywords: APJ, apelin, Goq ${ }_{16}$, GPCR, HTS, assay

\section{Introduction}

The apelin receptor (APJ) was cloned in 1993 as an orphan G-protein coupled receptor (GPCR) during the course of the Human Genome Project. ${ }^{1}$ The human APJ gene is located on the long arm of chromosome 11 and encodes a 377 amino acid G protein-coupled receptor. ${ }^{1}$ The gene for APJ was designated angiotensin-receptor like 1 (AGTRL1) due to sequence similarities between the two receptors. ${ }^{1}$ However, none of the known peptidergic ligands for the angiotensin receptors, including angiotensin, activate APJ. APJ remained an orphan GPCR until 1998 when the peptide Apelin was identified as its endogenous ligand. ${ }^{2,3}$ Over the years, apelin has emerged as an important regulator of various physiological processes. Both apelin and APJ are expressed in the central nervous system (CNS) and peripherally in a number of tissues. ${ }^{4,5}$ Expression of APJ has been noted within the vasculature of some organs and is a potent regulator of related processes including angiogenesis and vasoconstriction. ${ }^{6,8} \mathrm{APJ}$ is also expressed in nonvascular cell types in heart, liver, and CNS where its primary role is currently under investigation. ${ }^{5}$ Apelin and APJ are often co-localized within the same organ, 
suggesting an autocrine regulation of the receptor by its ligand. However, apelin has since been detected in blood suggesting that concomitant paracrine regulation of the receptor is also possible. ${ }^{9}$

The Apelin-APJ system has been implicated as a regulator of various physiological functions and is believed to play an important role in thermoregulation, immunity, glucose metabolism, angiogenesis, fluid homeostasis, cardiac function, hepatic function and renal function. ${ }^{9}$ APJ also acts as a co-receptor during HIV infection. ${ }^{10,11}$ Expression of apelin and APJ are either up- or downregulated in various pathophysiological conditions. In particular, the APJ appears to be an emerging target for the treatment of cardiovascular failure, liver fibrosis, cancer, angiopathies, pancreatitis, and as a prophylactic against HIV infection..$^{9,12}$ Unfortunately, small molecule ligands of the APJ having suitable pharmacological properties are lacking. Only one nonpeptide ligand has been reported to date. ${ }^{13}$ Therefore, additional chemotypes that can serve as templates for preclinical drug/probe development are needed. Past studies

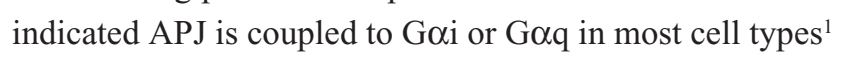
and activation of this receptor leads to suppression of cyclic adenosine monophosphate (cAMP; Gi-mediated) or activation of phospholipase $\mathrm{C}$ (PKC; Gq-mediated). To date, most in vitro experiments involving functional evaluation of APJ have utilized conventional radioligand binding assays and GTP $\gamma$ S assays, which are inconvenient due to the use of radioactivity, limited in throughput, and expensive. However, most GPCRs including APJ can be coupled to induce intracellular calcium mobilization via co-expression with the promiscuous $\mathrm{G} \alpha \mathrm{q}_{16}$ protein. ${ }^{5,14}$ This provides a convenient and robust microplate-based format to assay the function of APJ ligands using fluorescent calcium sensitive probes. In this paper we report the development and implementation of a calcium mobilization based HTS assay for APJ, which can be used to identify new ligands for this important receptor through an HTS campaign.

\section{Materials and methods Creation of APJ-G $\alpha q 16$ cells}

All cells were maintained in Dulbecco's modified Eagle's medium (DMEM)/F12, supplemented with 10\% fetal bovine serum (FBS), 1\% penicillin and streptomycin (p/s). Cell culture supplies were from Sigma Chemical Company (St. Louis, MO) unless otherwise specified. A plasmid expression construct harboring the cDNA for human AGTRL1 gene was obtained from University of Missouri at Rolla. The cDNA for AGTRL1 was re-cloned into a modified
pcDNA-3.1 mammalian expression vector (Invitrogen, Carlsbad, CA) by inserting the fragment in frame with a pre-prolactin (PPLS) leader sequence and an influenza hemagglutinin (HA) tag (Kind gift of Dr Brian Gilmour, RTI International, Research Triangle Park, NC). The plasmid construct (PPLS-HA-AGTRL1) was verified by sequencing. Chinese hamster ovary $(\mathrm{CHO})$ cells expressing $\mathrm{G \alpha q}_{16}$ were obtained from MDS Analytical Technologies (Concord, ON) and transfected with PPLS-HA-AGTRL1 using Fugene HD. Transfected cells were plated and selected using G418. A total of 64 clones were isolated. The clonal cells $\left(\mathrm{APJ}-\mathrm{G \alpha q}_{16}\right)$ were grown to confluence, and expression of functional APJ was tested using Apelin-13 peptide (Sigma) in a 96-well plate calcium mobilization assay as described below.

\section{Analysis of APJ expression using flow-cytometry}

Some clonal cell lines that were found to be functionally active using the calcium mobilization assay were also analyzed using flow-cytometry to confirm expression of HA-APJ protein. Approximately, $0.5 \times 10^{6}$ cells of each APJ-G $\alpha q_{16}$ clone and parental $\mathrm{CHO}-{\mathrm{G} \alpha q_{16}}_{1}$ cells were stained with an anti-HA monoclonal antibody (Sigma) at $1 \mu \mathrm{g} / \mathrm{mL}$ concentration or with a monoclonal anti-APJ antibody (R\&D Systems, Minneapolis, MN) at $2.5 \mu \mathrm{g} / \mathrm{mL}$ for $1 \mathrm{~h}$ on ice. Following three washes in phosphate-buffered saline (PBS), cells were stained again with an anti-mouse secondary antibody conjugated to Alexa-488 fluorophore for $30 \mathrm{~min}$ on ice. Cells were washed three additional times in PBS and immediately analyzed using flow-cytometry (C6 Flow Cytometer; Accuri, Ann Arbor, MI) in the FL1 (FITC) channel.

\section{Calcium mobilization assay}

Cells stably expressing APJ-G $\mathrm{Gq}_{16}$ were removed from their flasks using the nonenzymatic cell-stripper (Mediatech Inc., Orlando, FL) and quenched with DMEM/F12, 10\% FBS, centrifuged and re-suspended in the serum-containing media. Cells were counted with a hemocytometer and 30,000 cells were transferred to each well of a black Costar 96-well optical bottom plate (Corning Corporation, Corning, NY). Each plate was incubated at $37^{\circ} \mathrm{C}$ for $24 \mathrm{hr}$ to confluence. The culture media was removed from the plates and cells were subsequently loaded with a fluorescent calcium probe (Fluo-4 AM dye; Invitrogen/Molecular Probes, Eugene, OR) at a final loading concentration of $2 \mu \mathrm{M}$ in an HBSS-based buffer containing $20 \mathrm{mM}$ HEPES, $1 \%$ BSA and $10 \mu \mathrm{M}$ probenecid (Sigma) in a total volume of $225 \mu \mathrm{L}$. Cells were incubated at $37^{\circ} \mathrm{C}$ for $1 \mathrm{~h}$ and then stimulated with synthetic Apelin-13 
peptide (Sigma) at various concentrations using a Flexstation plate-reader, which automatically added the agonist at $10 \times$ concentration to each well after reading baseline values for $\sim 17 \mathrm{sec}$. Agonist-mediated change in fluorescence (488 nm excitation, $525 \mathrm{~nm}$ emission) was monitored in each well at $1 \mathrm{sec}$ intervals for $60 \mathrm{sec}$ and reported for each well. Data were collected using Softmax version 4.8 (MDS Analytical Technologies) and analyzed using Prism software (GraphPad, La Jolla, CA). Nonlinear regression analysis was performed to fit data and obtain maximum response $\left(\mathrm{E}_{\max }\right), \mathrm{EC}_{50}$, correlation coefficient $\left(r^{2}\right)$ and other parameters. Similar experiments were performed with other peptide agonists of the APJ as described in Table 1. All other apelin peptides were purchased from Anaspec Corporation (Fremont, CA). All experiments were performed 3-6 times to ensure reproducibility and data reported as mean \pm standard error, unless noted otherwise. The response of the selected APJ clonal cell line in the calcium flux assay was stable over 20 passes as determined through $\mathrm{EC}_{50}$ and $\mathrm{E}_{\max }$ values during experimentation.

\section{Z-factor determination}

Cells were plated at 30,000 cells per well in black 96-well plates and incubated at $37^{\circ} \mathrm{C}$ for $24 \mathrm{~h}$ until confluent. Cells were loaded with Fluo-4 dye and incubated for 60 minutes. Cells were stimulated with an Apelin-13 or buffer only control that was added via automation using the FlexStation instrument as described previously. The average and the standard deviation within each group were calculated. This experiment was repeated on three separate days and the $\mathrm{Z}$ factor ${ }^{15}$ was calculated with the following equation:

$$
\mathrm{Z}=1-\left(3 \mathrm{SD}_{\text {agonist }}+3 \mathrm{SD}_{\text {control }}\right) / \mid\left(\text { Mean }_{\text {agonist }}-\text { Mean }_{\text {control }}\right) \mid
$$

\section{Tolerance to solvents}

Tolerance of the assay to commonly used solvents DMSO and ethanol was determined by running the calcium mobilization assay at concentrations of $0.25 \%, 0.5 \%$ and $1 \%$ final concentration of solvent in the calcium mobilization buffer described above. Cells were subsequently stimulated with $10 \mu \mathrm{M}$ Apelin-13 in buffer containing either one of the solvents, or a mixture of both, and $\mathrm{E}_{\max }$ values were determined using the calcium mobilization assay. As controls, Apelin-13 peptide dissolved in water alone was used and cells stimulated with buffer alone (without solvents) as a negative control were also included.

\section{Screening of a pilot library}

A proprietary library of compounds available at RTI International was used for assay validation. Approximately 500 compounds directed towards GPCRs were used for this purpose. All compounds were screened at $10 \mu \mathrm{M}$ final concentration in duplicate along with Apelin-13 as a control in outside columns as agonists. Compounds that increased fluorescence to $\sim 50 \%$ of the Apelin- 13 control were deemed positive and re-screened. Concentration-response experiments were performed with positive compounds and the compounds were counter-screened using parental ${\mathrm{G} \alpha q_{16}}_{1}$ cells to rule out false positives.

\section{Results}

\section{Creation of CHO-Goql 6 cells} expressing APJ protein and development of a calcium mobilization assay for APJ

The overall goal of this project was to develop an HTS assay for the APJ that would expedite the discovery of novel ligands. APJ was chosen because of its potential impact as an important therapeutic target for various diseases. ${ }^{1}$ Past studies indicate that signaling through APJ is coupled primarily to Goi but this receptor can also signal through Goq, which provides an opportunity to monitor intracellular calcium flux as a measure of receptor activity. ${ }^{1}$ With these factors in mind, a heterologous system was designed in which APJ was coupled to the promiscuous Goq subunit $\mathrm{Goq}_{16}$ in $\mathrm{CHO}$ cells. Parental $\mathrm{CHO}-{\mathrm{G} \alpha q_{16}}_{1}$ cells were transfected with a

Table I EC ${ }_{50}$ determination of apelin peptides

\begin{tabular}{|c|c|c|}
\hline Peptide name & Peptide sequence & $\mathrm{EC}_{50} \pm \mathrm{SEM}[\mathrm{nM}]$ \\
\hline [Pyr I]-Apelin-13 & Pyroglutamate-RPRLSHKGPMPF-OH & $0.20 \pm 0.02$ \\
\hline Apelin-12 & H-RPRLSHKGPMPF-OH & $0.18 \pm 0.04$ \\
\hline Apelin-13 & H-QRPRLSHKGPMPF-OH & $7.82 \pm 0.69$ \\
\hline Apelin-17 & H-KFRRQRPRLSHKGPMPF-OH & $1.56 \pm 0.11$ \\
\hline Apelin-36 & H-LVQPRGSRNGPGPWQGGRRKFRRQRPRLSHKGPMPF-OH & $2.69 \pm 0.26$ \\
\hline FI3A-Apelin & H-QRPRLSHKGPMPA-OH & $202.17 \pm 12.93$ \\
\hline
\end{tabular}

Abbreviations: $\mathrm{EC}_{50}$, median effective concentration; SEM, standard error of mean. 
mammalian expression vector for APJ in which the gene for APJ, AGTRL1 was fused in frame to PPLS and HA epitope at the N-terminus. Addition of the PPLS improved processing of the GPCR and transport to the plasma membrane while the HA tag aided in specific detection of the protein. Following selection in antibiotic-containing media, clones were isolated and tested for expression of APJ using an automated intracellular calcium mobilization assay. Stimulation with an APJ agonist resulted in coupling of this receptor to $\mathrm{G \alpha q}_{16}$ and mobilization of intracellular calcium through PLC- $\beta .^{5}$ The fluorescent calcium indicator Fluo4-AM was used to monitor calcium mobilization in the clonal cell-lines. Almost $85 \%$ of the clones responded to Apelin-13 in varying degrees. A single clone was selected for further experimentation based on reproducible low $\mathrm{EC}_{50}$ and high $\mathrm{E}_{\max }$ values. In Figure 1A, the characteristic response of the selected clone to Apelin-13 peptide or to a related peptide in which the last amino acid residue is altered to A from $\mathrm{F}$ (F13A-Apelin) is demonstrated. The $\mathrm{EC}_{50}$ of this $\mathrm{APJ}-{\mathrm{G} \alpha \mathrm{q}_{16}}_{\text {clone was }}$ determined to be $\sim 7 \mathrm{nM}$ with Apelin-13, suggesting that the coupling of APJ to $\mathrm{G \alpha q}_{16}$ was very efficient. Surface expression of APJ protein was confirmed in the clonal cell-line and compared to parental $\mathrm{CHO}-{\mathrm{G} \alpha \mathrm{q}_{16}}_{16}$ cells through flow cytometry (Figure 1B) using two different monoclonal antibodies directed towards either the HA epitope or the APJ protein. The clonal cells expressing APJ demonstrated at least 10-fold more fluorescence compared to parental cells using either antibody. Further, detection of APJ-specific fluorescence with either antibody in nonpermeabilized cells indicated correct plasma membrane localization of the protein in agreement with past reports. ${ }^{1}$ However, the shift in fluorescence noted in APJ cells compared to parental cells was different with each antibody. This is possibly due to the difference in affinity and/or avidity of each antibody towards its respective target antigen. The anti-HA antibody is of extremely high quality and in our experience provides a more sensitive method of detection compared to the antiAPJ monoclonal antibody. In conclusion, clonal cell-lines co-expressing APJ and Goq16 were created to facilitate the designing of a functional HTS assay for this receptor.

\section{Comparison of different apelin peptides}

To further test the ability of the calcium mobilization assay to identify peptides that agonize APJ, we took advantage of the fact that while apelin is synthesized as a highly conserved prepropeptide (77 amino acids), ${ }^{16}$ which is then converted into various smaller peptides that differ in their pharmacological and physiological properties. ${ }^{1}$ The clonal APJ-G $\alpha q_{16}$ cell-line was

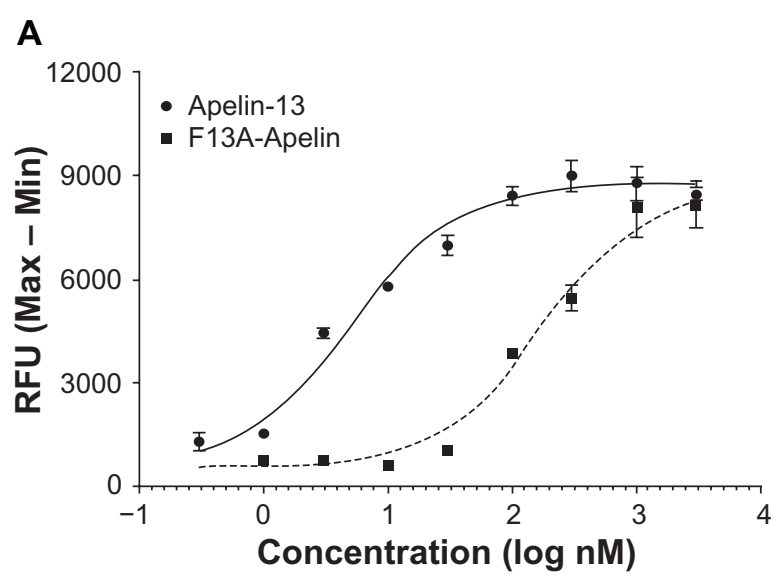

B
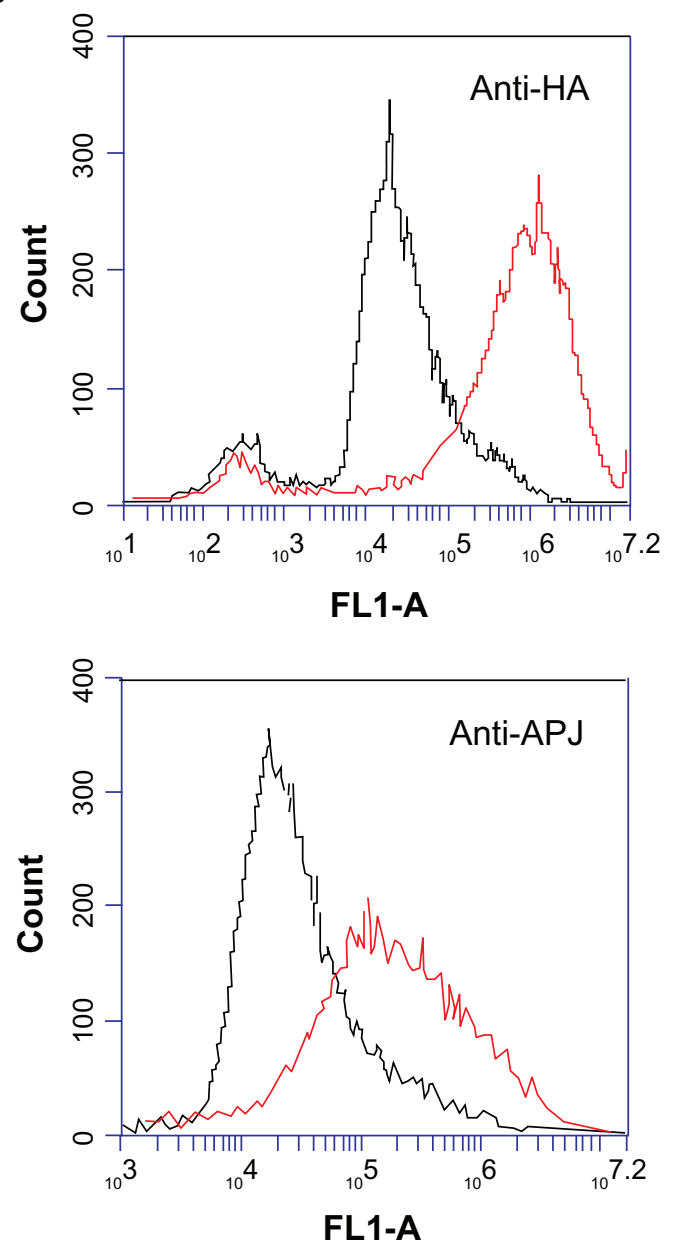

Figure I Creation and characterization of APJ-G $\alpha q_{16}$ cells. A) Calcium mobilization assay to monitor APJ function. APJ-G $\alpha$ q 6 cells were loaded with Fluo4-AM dye in a HBSS based buffer system and stimulated with various concentrations of Apelin- 13 (circle) or FI3A-Apelin (square) at various concentrations as described in 'Materials and Methods'. Nonlinear regression was used to fit fluorescence data. Both peptides acted as agonists at the receptor. B) Flow-cytometric characterization of APJ-G $\alpha q 16$ cells. Flow-cytometric analysis was performed with either parental $\mathrm{CHO}-\mathrm{G \alpha q}_{16}$ cells (black histogram) or APJ-G $\alpha q_{16}$ cells (red histogram). Nonpermeabilized cells were stained with an anti-HA (top) or anti-APJ (bottom) monoclonal antibody and secondary anti-mouse antibody conjugated to Alexa-488. The cells were analyzed as described in 'Materials and Methods'. APJ-G $\alpha q_{16}$ cells demonstrated significantly more fluorescence compared to parental cells. 
tested against several apelin variants as reported in Table 1. The most potent ligands demonstrated activities in the subnanomolar range at APJ. The values obtained were in line with reported experimental data. ${ }^{1}$ Thus a highly sensitive intracellular calcium mobilization assay was established for the APJ.

\section{Z-factor determination}

The calcium mobilization assay for APJ was tested for robustness, which is a requirement for HTS campaigns. The procedure outlined by Zhang and colleagues was used to generate a Z-factor for the assay. ${ }^{15}$ Apelin-13 was used to stimulate APJ activity, which was measured using the automated fluorescent calcium mobilization assay in a 96-well format. Cells stimulated with buffer only served as negative control for the assay. The assay was conducted in 96-well plates on three separate days for this purpose. The average fluorescence of Apelin-13 stimulated cells was 24476 arbitrary units with a standard deviation of 2384 arbitrary units. The average fluorescence of buffer only wells was 1321 arbitrary units with a standard deviation of 358 arbitrary units. As demonstrated in Figure 2, the assay was deemed to be highly robust and reproducible with a Z-factor of 0.64 . Thus, this assay is suitable for HTS applications.

\section{Effect of solvents on assay performance}

Two commonly used solvents, DMSO and ethanol, were tested for their compatibility with the APJ assay. Both of these solvents are routinely used as vehicles for test agents in laboratories and HTS campaigns. For these experiments, different concentrations of each solvent or a mixture of the two were tested. Concentration of the solvents was kept constant in all buffers used for stimulation. APJ activity was stimulated with Apelin-13 and the subsequent increase in fluorescence was measured. Comparison of $\mathrm{E}_{\max }$ values indicated that the assay was tolerant to either solvent alone or in a mixture to $1 \%$ total volume without a compromising loss in performance as demonstrated in Figure 3. Most commercial libraries are supplied as $10 \mathrm{mM}$ stocks and compounds are typically screened at $10 \mu \mathrm{M}$ final concentration. In the described assay, agonists were added at $10 \times$ concentration. Thus, a $1 \%$ final solvent concentration was deemed appropriate for assay development. Cells stimulated with buffer only without either solvent or Apelin-13 showed little change in fluorescence upon stimulation. The signal to noise ratio was $>10$ at all concentrations and combinations tested.

\section{Screening of a pilot library for assay validation}

A proprietary library of small molecule compounds enriched with GPCR ligands was used for assay validation. All compounds were screened in duplicate at $10 \mu \mathrm{M}$ final concentration as agonists. Data from a typical screening plate are demonstrated in Figure 4. Apelin-13 was used as a

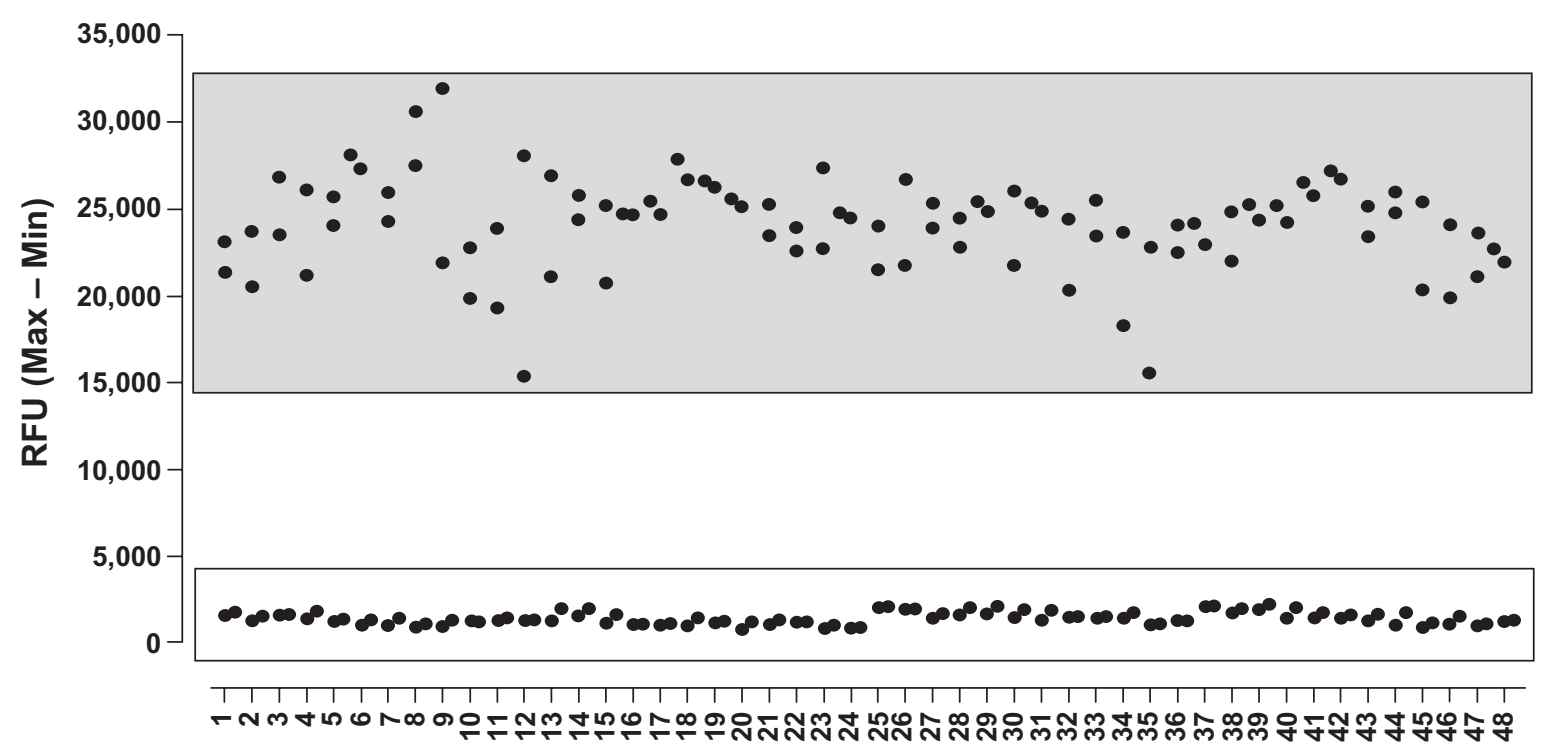

Well number

Figure 2 Z-factor determination. The Z-factor of the assay was determined by stimulating APJ-Goq ${ }_{16}$ cells with Apelin-13 (shaded gray) or buffer alone (shaded white) and measuring fluorescence increase induced by calcium mobilization as described in 'Materials and Methods'. Data from two separate $96-$-well plates run on different days are demonstrated. 


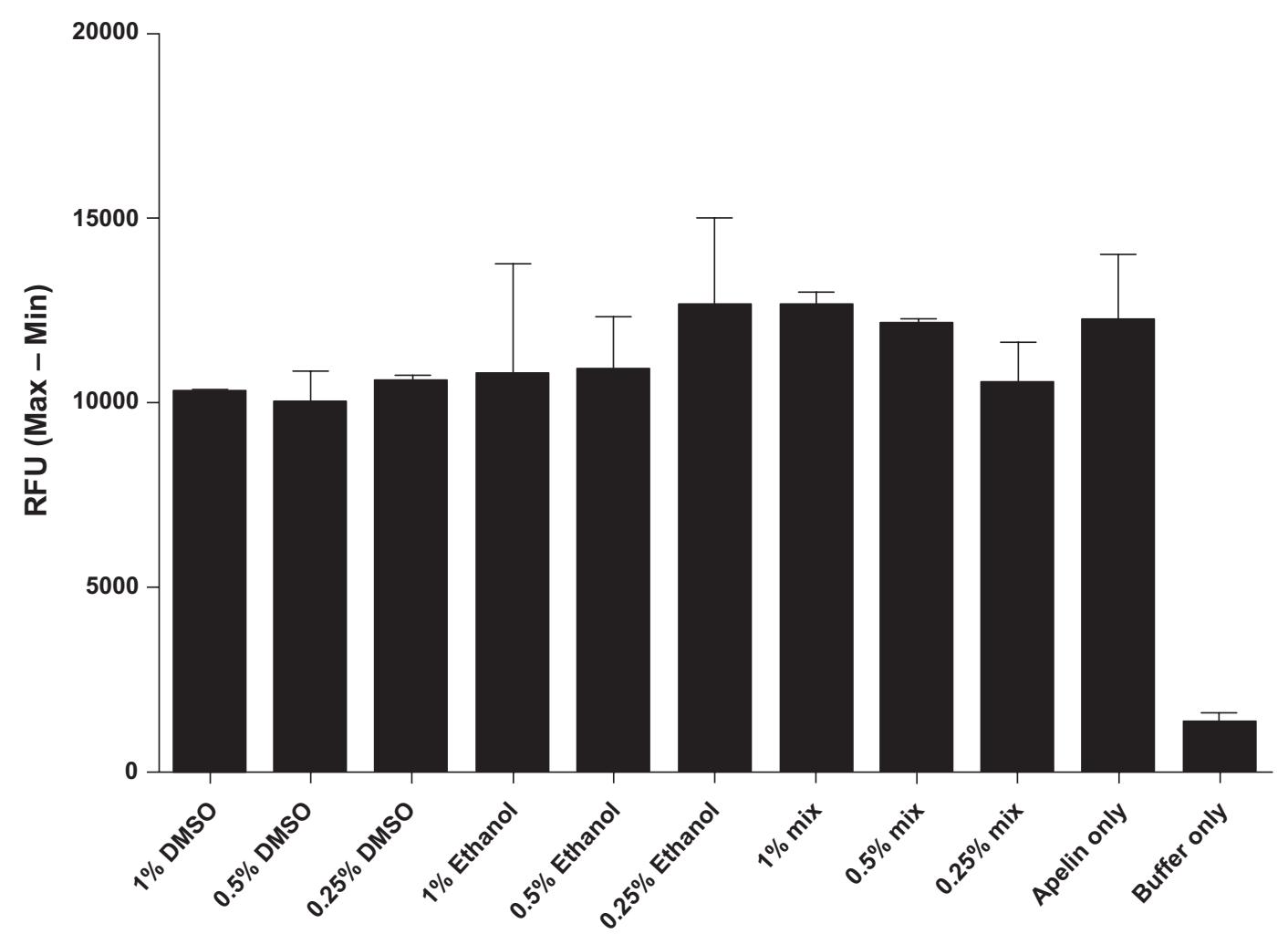

Figure 3 Tolerance of assay to solvents. The tolerance of the assay to DMSO, ethanol or a mixture of the two solvents was determined by stimulating APJ-G $\alpha q_{16}$ cells with various concentrations of the solvents as indicated in the figure and described under 'Materials and Methods'. Cells were stimulated with Apelin- 13 dissolved in water as vehicle for comparison. The assay performance is not critically affected up to $1 \%$ final concentration of each test vehicle or mixture.

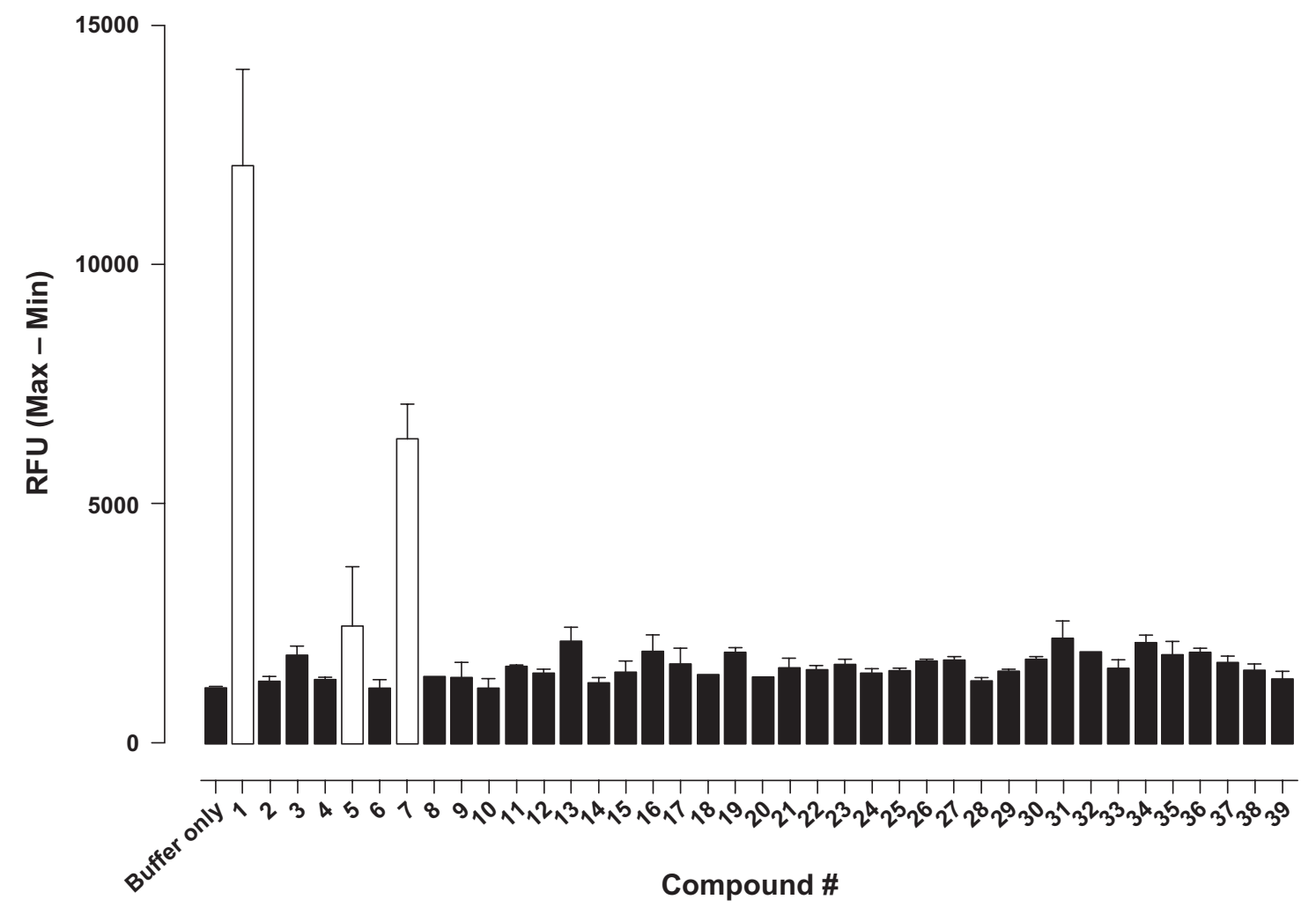

Figure 4 Screening of a pilot library. The assay was validated by screening a pilot library of compounds as described in 'Materials and methods'. Hits are indicated by clear columns. Data from a typical plate are reported. 
positive control for these experiments. The hit-rate was $\sim 0.5 \%$. Screening of the library yielded four structurally-related compounds that demonstrated agonism at the APJ. Three of the four hits are shown in Figure 4. The last hit was on a different plate and is not shown in the figure. Further, preincubation with these compounds abolished the response of the APJ to Apelin-13, possibly due to agonist-mediated desensitization (data not shown). Concentration-response experiments were performed subsequently to determine the $\mathrm{EC}_{50}$ value of the compounds identified through screening. The compounds were also counter-screened against parental ${\mathrm{G} \alpha q_{16}}_{1}$ cells to rule out false-positive hits that induced nonspecific mobilization of intracellular calcium. None of the compounds induced calcium mobilization in parental $\mathrm{G} \mathrm{q}_{16}$ cells. Representative concentration-response data with compound 1, which demonstrated strongest agonism towards APJ are demonstrated in Figure 5. These data validated the functional APJ assay and further consolidated its utility for HTS applications.

\section{Discussion}

Since its discovery in 1993, APJ has emerged as an interesting preclinical target for medications development due to its involvement in several important physiological processes. ${ }^{17}$ This receptor was cloned as an orphan GPCR and subsequently, Apelin was discovered as its endogenous ligand. Several research groups have now presented compelling evidence to suggest that this receptor might serve as a target to treat heart failure, cancer, angiopathies, HIV pathogenesis, liver fibrosis and other disorders. ${ }^{17,18}$ Only one nonpeptide ligand of the APJ has been reported to date, ${ }^{13}$ therefore the availability of a simple, rapid and robust assay to monitor activity of the APJ would serve to expedite the search for

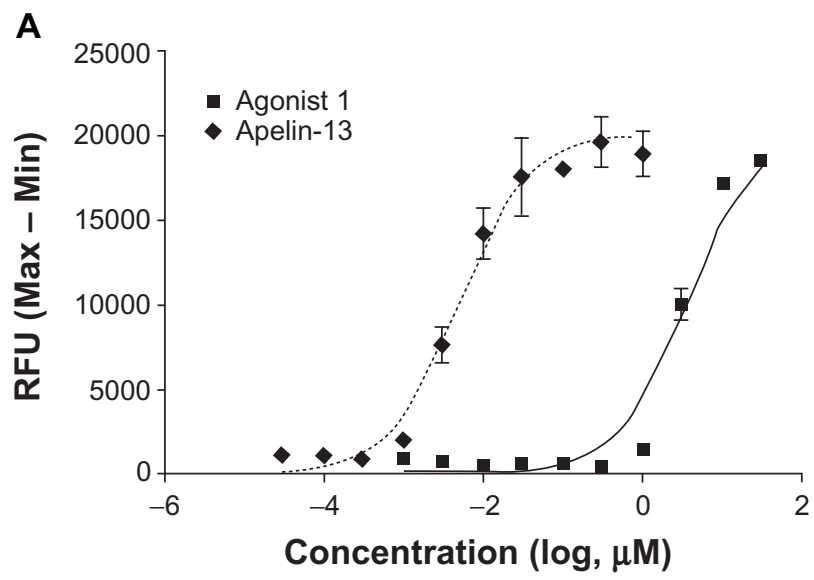

novel APJ ligands. With this in mind the goal of this project was to develop a validated APJ HTS-ready assay, which was lacking to date.

In this paper, development of a calcium mobilization assay for APJ is reported. Traditional assays for GPCR's including those for APJ are resource intensive, low in throughput and often involve radiometric detection. Past studies suggest that signaling through APJ is coupled to activation of Goi

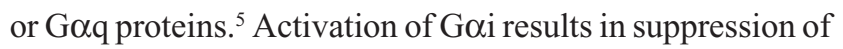
cAMP whereas activation of Goq through PLC can lead to mobilization of intracellular calcium. Excellent fluorescent probes of calcium are available commercially and can be used for automated assay development. Further, it has been demonstrated that several members of the GPCR family can be induced to signal through the promiscuous $\mathrm{G \alpha q}_{16}$ protein in a variety of cell types upon co-expression. ${ }^{14}$

Initially, APJ was engineered to be co-expressed in $\mathrm{CHO}$ cells already expressing $\mathrm{G \alpha q}_{16}$. Expression of the protein in clonal cells was verified using flow cytometry (Figure 1B). An automated fluorescent assay was subsequently developed to monitor signaling through this receptor coupled to $\mathrm{G} \alpha \mathrm{q}_{16}$ that produced a robust signal upon activation of the APJ by its cognate endogenous peptide ligand Apelin. Apelin itself is initially synthesized as a prepropeptide consisting of 77 amino acid residues, which is then cleaved into multiple shorter peptides with varying degrees of potency at the receptor. ${ }^{3}$ The last 13 amino acids of apelin (Apelin-13) are sufficient for full agonism of the receptor and this peptide has been extensively used in biological studies. ${ }^{19-22}$ Therefore, this peptide was used for assay development as demonstrated in Figure 1A. Studies were undertaken with various Apelin peptides to better characterize their response at the APJ (Table 1).

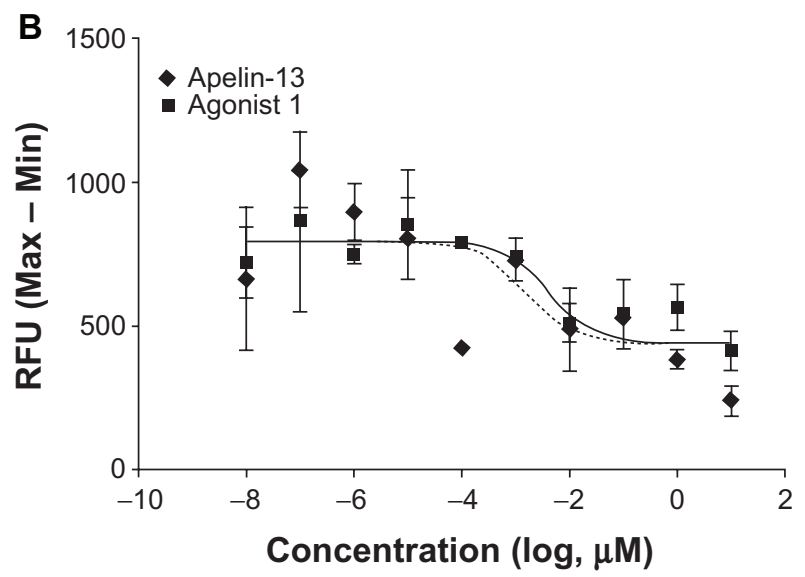

Figure 5 Validation of hits. Hits from screening a pilot library were verified by running concentration-response experiments in APJ-G $\alpha q_{16}$ cells or parental $\mathrm{CHO}-\mathrm{G}_{\mathrm{A}} \mathrm{q}_{16}$ cells as described under 'Materials and Methods'. A) Concentration-response studies with a typical agonist. Data from a typical concentration-response experiment are demonstrated

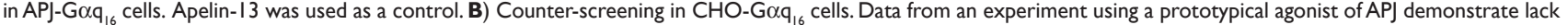
of response in parental $\mathrm{CHO}-\mathrm{G \alpha q}_{16}$ cells indicative of specific agonism at the APJ. Apelin- 13 failed to show a response and served as a negative control. 
Of the peptides tested, a pyroglutamate form of Apelin-13 (Pyr-Apelin-13), which is biologically more stable, was found to be a potent activator of this receptor with $\mathrm{EC}_{50}$ in the picomolar range. Apelin-36, which is a larger form of Apelin showed lower activity compared to Pyr-Apelin-13. A related peptide with an alanine residue instead of phenylalanine at the C-terminal of Apelin (F13A-Apelin) demonstrated reduced activity as an agonist in accordance with past in vitro data. ${ }^{5}$ Surprisingly, certain groups have reported that F13A-Apelin behaves as an antagonist in vivo. ${ }^{7,23}$ Additional studies will be required to resolve this discrepancy in the future. In general, data presented herein agree well with data reported from past studies with this receptor utilizing different in vitro methodologies. ${ }^{1,5}$ Thus, these studies indicated that coupling to $\mathrm{G} \alpha \mathrm{q}_{16}$ did not affect functional activation of the APJ and that a calcium flux assay was suitable for monitoring the function of this receptor in vitro.

The assay for APJ was subsequently tested and adapted for HTS application. The assay was deemed robust and reliable with a Z-factor of 0.6 (Figure 2). The assay performed well in buffers containing DMSO, ethanol, or a mixture of the two solvents to a final 1\% concentration (Figure 3 ). Finally, a small library of compounds was screened for assay validation. The hit-rate of the assay was $\sim 0.5 \%$ using a library enriched with GPCR ligands (Figure 4). The hits were further characterized using concentration-response experiments and counter-screened in parental $\mathrm{CHO}-\mathrm{G}_{\alpha q_{16}}$ cells to rule out false positive hits (Figure 5). Additional studies are currently underway to characterize the physiological influence of these compounds. Data related to their structure and biological activity will be reported in the future.

In theory, the assay reported in this paper can be used to identify several different types of APJ ligands including competitive antagonists, noncompetitive antagonists, inverse agonists and allosteric modulators. All types of antagonists will induce a rightward shift in the concentration response curve with Apelin-13 or another agonist upon pre-incubation. However, noncompetitive antagonists will typically produce lower $\mathrm{E}_{\max }$ values. Inverse agonists alone can be expected to reduce basal fluorescence of the cells in a concentration dependent manner. Allosteric modulators on the other hand should either increase or decrease $E_{\max }$ values upon stimulation with an agonist without inducing a significant shift in the concentration-response curves. A larger HTS campaign in the future might lead to the discovery of these different types of ligands for APJ. It also remains to be seen whether the assay can be used to detect endogenous levels of apelin in more complex biological fluids including blood, plasma or breast milk. These avenues are currently under investigation. In conclusion, a calcium mobilization assay has been developed for the APJ that is HTS amenable and can be used to identify ligands of this receptor that is rapidly emerging as a target for drug development to treat important disorders.

\section{Acknowledgments}

The authors would like to thank RTI International for supporting this project with internal $R \& D$ funding. The authors have no known conflicts of interest related to the project.

\section{References}

1. Carpene C, Dray C, Attane C, et al. Expanding role for the apelin/APJ system in physiopathology. J Physiol Biochem. 2007;63(4):359-373.

2. Lee DK, Cheng R, Nguyen T, et al. Characterization of apelin, the ligand for the APJ. J Neurochem. 2000;74(1):34-41.

3. Habata Y, Fujii R, Hosoya M, et al. Apelin, the natural ligand of the orphan receptor APJ, is abundantly secreted in the colostrum. Biochim Biophys Acta. 1999;1452(1):25-35.

4. De Mota N, Lenkei Z, Llorens-Cortes C. Cloning, pharmacological characterization and brain distribution of the rat apelin receptor. Neuroendocrinology. 2000;72(6):400-407.

5. Medhurst AD, Jennings CA, Robbins MJ, et al. Pharmacological and immunohistochemical characterization of the APJ and its endogenous ligand apelin. J Neurochem. 2003;84(5):1162-1172.

6. Kasai A, Shintani N, Oda M, et al. Apelin is a novel angiogenic factor in retinal endothelial cells. Biochem Biophys Res Commun. 2004;325(2):395-400.

7. Tiani C, Garcia-Pras E, Mejias M, et al. Apelin signaling modulates splanchnic angiogenesis and portosystemic collateral vessel formation in rats with portal hypertension. J Hepatol. 2009;50(2):296-305.

8. Hus-Citharel A, Bouby N, Frugiere A, et al. Effect of apelin on glomerular hemodynamic function in the rat kidney. Kidney Int. 2008;74(4):486-494.

9. Ladeiras-Lopes R, Ferreira-Martins J, Leite-Moreira AF. The apelinergic system: the role played in human physiology and pathology and potential therapeutic applications. Arq Bras Cardiol. 2008;90(5):343-349.

10. O'Donnell LA, Agrawal A, Sabnekar P, et al. Apelin, an endogenous neuronal peptide, protects hippocampal neurons against excitotoxic injury. J Neurochem. 2007;102(6):1905-1917.

11. Zou MX, Liu HY, Haraguchi Y, et al. Apelin peptides block the entry of human immunodeficiency virus (HIV). FEBS Lett. 2000;473(1):15-18.

12. Japp AG, Newby DE. The apelin-APJ system in heart failure: pathophysiologic relevance and therapeutic potential. Biochem Pharmacol. 2008;75(10):1882-1892.

13. Iturrioz $\mathrm{X}$, Alvear-Perez $\mathrm{R}$, De Mota $\mathrm{N}$, et al. Identification and pharmacological properties of E339-3D6, the first nonpeptidic apelin receptor agonist. FASEB J. 2009 Dec 29. [Epub ahead of print].

14. Kostenis E. Is Galpha16 the optimal tool for fishing ligands of orphan G-protein-coupled receptors? Trends Pharmacol Sci. 2001;22(11):560-564.

15. Zhang JH, Chung TD, Oldenburg KR. A simple statistical parameter for use in evaluation and validation of high throughput screening assays. J Biomol Screen. 1999;4(2):67-73.

16. Tatemoto K, Hosoya M, Habata Y, et al. Isolation and characterization of a novel endogenous peptide ligand for the human APJ. Biochem Biophys Res Commun. 1998;251(2):471-476.

17. Llorens-Cortes C, Moos F. Opposite potentiality of hypothalamic coexpressed neuropeptides, apelin and vasopressin in maintaining body-fluid homeostasis. Prog Brain Res. 2008;170:559-570. 
18. Kleinz MJ, Davenport AP. Emerging roles of apelin in biology and medicine. Pharmacol Ther. 2005;107(2):198-211.

19. Bai B, Tang J, Liu H, et al. Apelin-13 induces ERK1/2 but not $\mathrm{p} 38$ MAPK activation through coupling of the human apelin receptor to the Gi2 pathway. Acta Biochim Biophys Sin (Shanghai). 2008;40(4):311-318.

20. Guo L, Li Q, Wang W, et al. Apelin inhibits insulin secretion in pancreatic beta-cells by activation of PI3-kinase-phosphodiesterase 3B. Endocr Res. 2009;34(4):142-154.

21. Hosoya M, Kawamata Y, Fukusumi S, et al. Molecular and functional characteristics of APJ. Tissue distribution of mRNA and interaction with the endogenous ligand apelin. $J$ Biol Chem. 2000;275(28):21061-21067.
22. Seyedabadi M, Goodchild AK, Pilowsky PM. Site-specific effects of apelin-13 in the rat medulla oblongata on arterial pressure and respiration. Auton Neurosci. 2002;101(1-2):32-38.

23. Lee DK, Saldivia VR, Nguyen T, et al. Modification of the terminal residue of apelin-13 antagonizes its hypotensive action. Endocrinology. 2005;146(1):231-236.
International Journal of High Throughput Screening

\section{Publish your work in this journal}

International Journal of High Throughput Screening is an international, peer-reviewed, open access journal publishing original research, reports, editorials, reviews and commentaries dedicated to all aspects of high throughput screening, especially related to drug discovery and associated areas of biology and chemistry. The manuscript management sys-

\section{Dovepress}

tem is completely online and includes a very quick and fair peer-review system. Visit http://www.dovepress.com/testimonials.php to read real quotes from published authors.

Submit your manuscript here: http://www.dovepress.com/international-journal-of-high-throughput-screening-journal 